Facial recognition during early motherhood: Investigating the persistence of age and affect biases

Madison Bunderson ${ }^{\mathrm{a}}$, Kathryn Armstrong ${ }^{\mathrm{a}}$, Linda C. Mayes ${ }^{\mathrm{a}}$, Eamon J. McCrory ${ }^{\mathrm{b}}$, Chloe ThompsonBooth $^{\mathrm{b}}$, Helena J.V. Rutherford ${ }^{\mathrm{a}}$

aYale Child Study Center, 230 South Frontage Rd, New Haven, CT 06520, USA

bDivision of Psychology and Language Sciences, University College London, Gower Street, London, UK

Correspondences to:

Helena JV Rutherford, PhD Yale Child Study Center 230 South Frontage Road New Haven, CT 06520

USA

Phone: 203-737-3408

Fax: 203-785-7926

Email: helena.rutherford@yale.edu

\title{
Declarations of Interest
}

Conflicts of Interest: None Competing Interests: None

Funding: This work was supported by the Anna Freud Centre (UK). The funding source had no involvement in any part of the study design, writing of the manuscript or decision to submit the manuscript. 
Highlights

- Emotion facial recognition speed was assessed in mothers within one year postpartum

- Mothers responded to happy and sad infant and adult faces in a morph task

- The happy face advantage and the own-age bias were present in mothers

- Findings replicate the results of prior research during pregnancy 


\begin{abstract}
Converging evidence demonstrates increased levels of sensitivity to infant faces in mothers. This may be reflective of a series of psychological and neurobiological changes that occur in the transition to, and during, early parenthood for the purpose of appropriate caregiving; however, this enhanced infant facial recognition is in contrast with the general adult literature regarding facial processing. In the current study, we aimed to replicate a prior study of emotion facial recognition in pregnant women in a sample of mothers with children under a year old, utilizing a paradigm in which adult and infant faces gradually changed from neutral expressions to either happy or sad expressions. Mothers were faster at the recognition of adult faces in comparison to infant faces, and were also faster at happy faces in comparison to sad faces.

Results are discussed in context of the current processing literature regarding the perinatal period, and implications for the persistence of the own-age bias and happy face advantage are considered.
\end{abstract}

Keywords: motherhood; postpartum; facial recognition; emotion recognition; happy face advantage; own-age bias

Classification codes: 2300; 2323; 2340; 2956 


\section{Introduction}

The ability to process and understand the facial expressions of those around us is an integral part of daily human interactions (Darwin \& Prodger, 1998). It is one of the most important factors in our ability to communicate and interact with others (e.g., Fridlund, 1991). Its significance is further emphasized in the interactions between a caregiver with their infant, in which the use of facial expressions is one of the critical ways in which a young child is able to communicate (Ferrey et al., 2016). It has been suggested in previous research that in the transition to parenthood, parents-with research focusing primarily on mothers - undergo a series of psychological and neurobiological changes that prepare them for caregiving (e.g., Mayes, Rutherford, Suchman, \& Close, 2012). For example, converging evidence demonstrates increased levels of sensitivity to auditory and facial infant cues in mothers when compared to non-mothers (Proverbio, Brignone, Matarazzo, Del Zotto, \& Zani, 2006; Purhonen et al., 2001). These appropriate levels of responsiveness and sensitivity from a caregiver are essential to nurturing healthy development in the child (Barba-Müller, Craddock, Carmona, \& Hoekzema, 2019; Kim, 2016).

These shifts in sensitivity towards infant cues, particularly facial cues, may be at odds with patterns of behavior of typical adult face recognition. Two biases present in facial processing in the general population are the own-age bias and the happy face advantage (e.g., Kirita \& Endo, 1995; Rhodes \& Anastasi, 2012). The own-age bias is the tendency of having better memory for faces that are part of one's own age group in comparison to faces of another age group. Thus, as an adult, memory tends to be superior for adult faces, rather than child or infant faces (Rhodes \& Anastasi, 2012). This is thought to occur because individuals are most likely to have higher levels of contact and exposure, and thus more expertise, in the process of recognizing one's own age group (e.g., Kuefner, Macchi Cassia, Picozzi, \& Bricolo, 2008; Valentine, 1991). The second bias is linked to affect; it has been repeatedly observed that, when compared to sad faces, happy faces are processed more quickly and holistically. This is partially thought to be explained by the unique changes in facial features for happy faces in 
comparison to other emotional expressions, as happy features tend to have less overlap with other emotions (Johnston, Katsikitis, \& Carr, 2001) and can also stand alone as a representation of happiness (e.g., a smile; Adolphs, 2002). The happy face advantage follows a larger general trend, in which positive stimuli are more quickly categorized than negative stimuli, even in the case of words or nonsocial pictures (e.g., Matlin \& Stang, 1978).

However, it should be noted that a majority of facial processing research that establishes these biases primarily utilizes adult or adolescent faces. Less information is known overall about the processing of infant faces, despite the fact that infant faces differ structurally from other age categories. Conceptualized by Lorenz $(1943,1971)$ as Kindchenschema, or baby schema, infant facial features tend to be configured with larger heads, more expansive foreheads, bigger eyes, and more rounded cheeks. These features overall are typically perceived as more cute and attractive, thus prompting a differing response in the general population in comparison to adult faces (e.g., Endendijk, Spencer, van Baar, \& Bos, 2018; Glocker et al., 2009; Lobmaier, Sprengelmeyer, Wiffen, \& Perrett, 2010). Therefore, as the structure of, and the response elicited by, an infant face differs in comparison to an adult face, the examination of facial processing as it pertains specifically to infant faces, rather than the adult faces typically used in the general literature, warrants further investigation.

Due to the aforementioned changes that happen during the transition into parenthood (e.g., Hoekzema et al., 2017; Kim, Strathearn, \& Swain, 2016; Mayes et al., 2012) and the differential features of infant faces, previous research has investigated how two of the consistent biases in facial processingthe own-age bias and the happy face advantage - may or may not differ during early parenthood or when infant, in comparison to adult, faces are used. There is some evidence that mothers, in comparison to non-mothers, have greater attentional engagement with emotional expressions and infant faces. These cues are thought to be biologically salient to the mother while also generating an incentive for caregiving behaviors (e.g., Ferrey et al., 2016; Thompson-Booth et al., 2014a; Thompson-Booth et al., 2014b). This maternal bias towards infant emotion has also been found at a neurobiological level, with maternal 
response differing in regards to infant crying in comparison to laughter (Seifritz et al., 2003). The findings of these studies suggest that increased attentional allocation to infant distress may be an essential part of the parent-child interaction, as it allows the mother to prioritize relevant infant signals and subsequently react in a sensitive and appropriate way to resolve the source of distress (ThompsonBooth et al., 2014b). Attention to distress is important not only in the immediate moment of caregiving, but also in the formation of long-term mother-child attachment and overall development of the child (e.g., Ainsworth, 1979; Goldberg, Grusec, \& Jenkins, 1999; McElwain \& Booth-LaForce, 2006).

Building upon this research, Byrne et al. (2019) investigated whether or not differences in facial age and affect recognition occurred in pregnant women viewing infant and adult faces. To address this, an experimental paradigm in which faces slowly morph from a neutral expression to a happy or sad expression was employed. Facial morphing tasks such as this have been widely employed to measure emotion recognition (e.g., Joormann \& Gotlib, 2006; Lowyck et al., 2016). Byrne et al., (2019) found that pregnant women were faster and more accurate at the recognition of adult faces and happy faces (across infant and adult faces), with the fastest and most accurate category being adult happy faces, and the slowest and least accurate category being sad infant faces (Byrne et al., 2019). Although these findings are supported by the general adult literature (i.e., own-age bias and happy face advantage; Kirita \& Endo, 1995; Rhodes \& Anastasi, 2012), research around maternal attention allocation literature would suggest that a mother would be the fastest and most accurate at recognizing sad infant faces. Further understanding of maternal facial processing is critical, as emotion recognition may provide a mechanism by which mothers are able to respond appropriately and sensitively to the needs of their infant. This communication process is integral to the formation of a robust and healthy attachment between mother and infant, and evidence overwhelmingly indicates that healthy attachment is one of the most important factors in a child's development (e.g., Bowlby, 1997; Snyder, Shapiro, \& Treleaven, 2012).

Given the significance of maternal facial processing, and in light of the potential implications for later development, the current study investigates and seeks to replicate the aforementioned findings of 
Byrne et al. (2019). As Byrne et al. (2019) employed a sample of women in pregnancy, the goal of the current study is to extend usage of the same facial morph paradigm in order to examine facial affect recognition in an independent sample of recent mothers. We present competing hypotheses. On the one hand, in light of recent evidence (Byrne et al., 2019), we hypothesized that mothers will more quickly recognize affect in both own-age and happy faces, patterns which are consistent with the general adult literature. On the other hand, given the increased saliency of infant cues to mothers, particularly infant distress, we hypothesized that mothers may also demonstrate faster recognition of affect in sad infant faces.

2. Methods and Materials

2.1. Participants

70 mothers, independent of the sample utilized in Byrne et al. (2019), participated in the current study. All participants were recruited through the Yale Baby School (YBS) database, which is an educational research collaborative that recruits women who have recently given birth in local community hospitals (https://babyschool.yale.edu/). The women completed the current task as part of an introductory visit to the YBS. The women $(M$ age $=32.59$ years, $S D=7.168)$ classified their ethnicity as either nonHispanic $(n=58)$ or Hispanic $(n=12)$. A majority of women $(n=61)$ were either married or in a domestic partnership. As an estimate for socioeconomic status, all mothers also reported their highest level of education; 6 mothers had completed a high school diploma or less and 63 completed some form of higher education past a high school diploma, with data missing on 1 mother. The sample consisted of both first-time $(n=24)$ and experienced $(n=46 ; M$ children $=2.39$; range of 2 to 6$)$ mothers. The mothers also reported the length of the postpartum period since their most recent child $(M=2.90$ months; $S D=4.53)$ and whether their most recent child was female $(\mathrm{n}=32)$ or male $(\mathrm{n}=38)$. Information on feeding mode was also collected, with 41 mothers solely breastfeeding, 14 solely formula feeding, and 15 doing a combination of both. A full breakdown of all demographic information collected 
on the sample is presented in Table 1. Yale School of Medicine's Human Investigation Committee approved all procedures prior to recruitment. All participants consented to participation and were compensated for their involvement.

Characteristic $n(\%)$

Maternal Age (years), mean $\pm S D$

$32.59 \pm 7.168$ 
Ethnicity

Non-Hispanic

Hispanic

Relationship Status

Single

Never Married

Married or in domestic partnership

Divorced

Education Level

Some high school

High school diploma

Vocational or trade school

Some college

Associate's degree

Bachelor's degree

Master's degree

Bachelor's or master's degree

Doctoral degree

N/A

Parity

Primiparous

Multiparous, mean; range

Postpartum Period (months), mean $\pm S D$

Hours with Infant, mean $\pm S D$

Sex of Infant

Male

Female
$58(83 \%)$

$12(17 \%)$

$7(10 \%)$

$2(3 \%)$

$59(84 \%)$

$2(3 \%)$

$1(1 \%)$

$5(7 \%)$

$2(3 \%)$

$10(14 \%)$

$4(6 \%)$

$5(7 \%)$

$10(14 \%)$

$22(31 \%)$

$10(14 \%)$

$1(1 \%)$

$24(34 \%)$

$46(66 \%), 2.39 ; 2-6$

$2.90 \pm 4.53$

$22.29, \pm 3.72$

$38(54 \%)$

$32(46 \%)$ 
Feeding Mode

Breastfeeding

$41(59 \%)$

Formula feeding

$14(20 \%)$

Both breast and formula feeding

$15(21 \%)$

Table 1. Demographic characteristics for all participants $(n=70)$.

\subsection{Design}

Procedure: All procedures were completed within a designated research room. As part of the broader visit, participants first viewed an oral presentation of the YBS and completed consent forms. They then completed demographic measures and self-report questionnaires. Following the self-report questions, participants completed a facial morph task. The task has been utilized and described in full detail in previous studies (e.g., Byrne et al., 2019). The facial morph task consists of the transformation of a neutral expression into either a happy or sad expression, for both adult and infant faces. Participants read the on-screen instructions themselves and were also offered the opportunity to have the instructions read aloud to them for clarification before beginning the task. During the task, they selected buttons on the screen labelled "happy" or "sad" as soon as they were able to recognize the emotion expressed by the face. Participants were able to change their response as many times as desired, and could do so until the full facial emotion appeared. The participants had a practice trial to familiarize themselves with the task requirements while a research assistant monitored for participant understanding. The entire facial morph task took approximately 10 minutes to complete.

Face Stimuli Description: The stimuli were presented on a Dell Optiplex 7010 computer, using a 17-inch LCD monitor at a screen resolution of $1280 \times 1024$. The experiment was run using E-Prime 2.0 (Schneider, Eschman, \& Zuccolotto, 2012). There were four morph conditions (happy adult, sad adult, happy infant, and sad infant) with 14 trials each for a total of 56 trials. Seven different adult and seven 
different infant facial identities were randomly presented. The morphs were created utilizing MorphAge software (MorphAge version 4.0, Creaceed, at http://www.creaceed.com). The NimStim Set of Facial Expressions (Tottenham et al., 2009) provided the adult face stimuli. The NimStim models (closed mouth) used for this experiment were $01,02,09,23,24,34$, and 36 . Infant faces were obtained from a former study by Strathearn and McClure (2002). All models were Caucasian and unfamiliar to the participant. To address potential intensity level differences between each of the full-blown emotional expressions, 24 nulliparous females (mean age 24 years; SD 3 years) rated each full-blown emotional face (happy, sad, infant, adult) employing a scale from "1" - "not at all intense" to "7" "very intense". Happy faces $(M=4.36 ; S D=0.60)$ were rated as less intense than sad $(M=5.07 ; S D=0.72)$ faces, $t(23)$ $=-6.23, p<001$. Infant $(M=4.82 ; S D=0.75)$ and adult $(M=4.61 ; S D=0.58)$ faces were rated as comparable in their intensity, $t(23)=-1.69, p=.10$.

Faces were presented in grey scale inside of a black box, located centrally on a white screen. All faces were approximately $8 \mathrm{~cm} \times 11 \mathrm{~cm}$. After the initial face presentation, the participant had to click to begin the morphing. Once started, the morph involved 20 total frames, with 500ms allotted per frame. Each frame increased emotional expression in 5\% increments, until 100\% expression was presented and the trial ended. In total, each trial took at least $10,000 \mathrm{~ms}$, but time could be increased by either waiting to click to begin the trial, or by waiting to make a response until the complete facial expression appeared. An example presentation of the adult happy condition is presented in Figure 1.

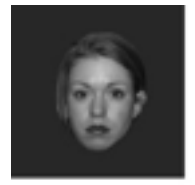

Initial Face
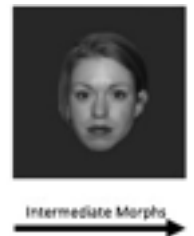

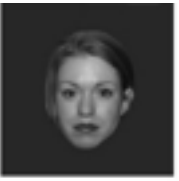

Morph 7

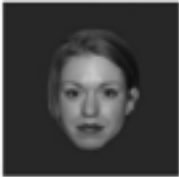

intermedine varnts

Morph Trial Sequence

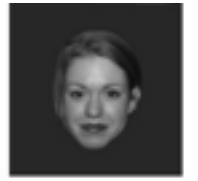

Morph 14

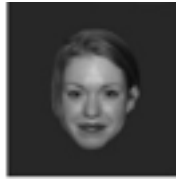

intersediate Mough

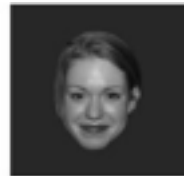

Final Face

Figure 1. Examples of the face stimuli for a trial in the adult happy condition. Faces here are shown at the initial frame, frame 7 , frame 14, and the final frame, with intermediate morphs also presented. Participants respond as soon as they recognize the emotional expression. 


\subsection{Self-Report Measures}

Demographic Questions: Participants began by reporting a variety of demographic information about themselves, as represented in Table 1.

Generalized Anxiety Disorder, 7-item: The GAD-7 (Spitzer, Kroenke, Williams, \& Löwe, 2006) was completed by participants before the task. The GAD-7 is a 7-item self-report measure of anxiety symptoms that have been experienced in the last two weeks. Respondents report how much each symptom has bothered them on a scale of "not at all" to "nearly every day", and can score between 0 (very little anxiety) and 21 (severe anxiety). Participant levels of anxiety can be classified as mild (5-9), moderate (10-14), or severe (15-21). This measure has been well-tested and established as a reliable measure of anxiety symptomology (Spitzer et al., 2006). The mean score for the GAD-7 was 2.68 (median $=2.00, S D=3.13$ ), indicating overall low levels of anxiety in this sample.

Patient Health Questionnaire, Short Form: The PHQ-2 (Kroenke, Spitzer, \& Williams, 2003) was completed by participants before the task. The PHQ-2 is a 2-item self-report measure of depressive symptoms that have been experienced in the previous two weeks. Participants report how much each problem has bothered them on a scale of "not at all" to "nearly every day", and can score between 0 (minimal depression) and 6 (serious depression). Participants are considered to have a positive screen when they score a 3 or above. This measure has been thoroughly researched for both reliability and validity in the measurement of depression (Kroenke et al., 2003). The mean score for the PHQ-2 was 0.38 (median $=0.00, S D=0.74$, , indicating minimal levels of depression in this sample.

\subsection{Data Analysis}

4 women were excluded from data analysis due to either incompletion of the task $(n=1)$, situational distraction such as a fussing infant $(n=2)$, or as an extreme outlier based on their reaction time during the task $(n=1)$. There were no demographic differences between excluded mothers and those included in the analysis. Data from a total of 66 women was then analyzed. 
Mean reaction times (RTs) were calculated in milliseconds (ms) for the four morph conditions: happy adult, sad adult, happy infant, and sad infant. Only the participant's initial response was included in the analysis. The mean accuracy for each response was also calculated for the participant's first response as a proportion, in which a score of 1.00 is equivalent to $100 \%$ accuracy across all trials within a condition. Only trials with correct responses for each condition were included in analyses. RTs were not normally distributed and were log transformed for analysis purposes. Log transformation improved the skewness and distribution of the data, justifying this approach.

For the main analysis, mean log-transformed RT was analyzed using a $2 \times 2$ repeated measures ANOVA, with within-subjects factors of facial age (adult vs. infant) and facial affect (happy vs. sad). Partial eta squared ( $\eta 2$ ) was also calculated to examine effect size, where .01 represents a small effect size, .06 represents a medium effect size, and .14 represents a large effect size (Cohen, 1988).

\section{Results}

\subsection{Preliminary Analysis}

Accuracy was highest for adult happy faces $(M=.99, S D=.03)$, followed by adult sad $(M=.98$, $S D=.04)$, infant happy $(M=.97, S D=.06)$, and infant sad faces $(M=.83, S D=.15)$. To be more representative of the data analyzed, Figure 2 presents the anti-log of the mean RTs for correct trials for each condition. 


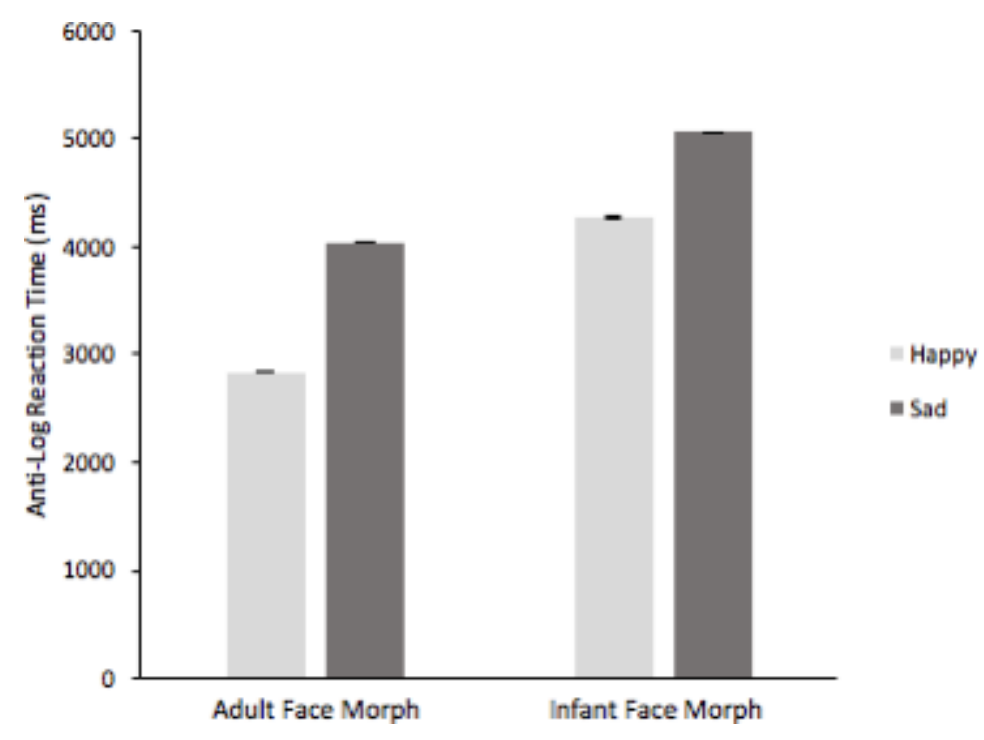

Figure 2. Mean maternal anti-log RTs for initial emotion recognition in adult and infant happy and sad face morph trials. Error bars represent standard error of the mean anti-log RT.

After log transformation, bivariate correlations between participants' RTs for each morph condition and demographic variables were examined. None of the demographic variables were associated with one another. Furthermore, when controlled for multiple comparisons, none of the demographic factors were correlated with participant RTs. Our depression and anxiety measures were associated with one another $(r=.27, p=.03)$, but were also not correlated with participant RTs.

\subsection{Main Analyses}

Reaction Time: A 2 × 2 repeated measures ANOVA revealed a main effect of facial age on RT, where participants were faster at recognition of adult faces than infant faces, $F(1,65)=330.86, p<.001$, $\eta 2=.84$. In addition, there was also a main effect of facial affect, with participants more quickly recognizing happy faces rather than sad faces, $F(1,65)=313.69, p<.001, \eta 2=.83$. Facial age and affect also interacted, $F(1,65)=66.83, p<.001, \eta 2=.51$. As evidenced in Figure 2, the interaction appears to be driven by a greater difference in recognition speed for happy and sad adult faces in comparison to the smaller difference in recognition speed for happy and sad faces in the infant condition. 
To test this interpretation, we examined the difference in response times (sad minus happy) between the infant and adult conditions, finding that the difference was statistically larger for adult faces as compared to infant faces, $t(65)=8.18, p<.001$. We further examined whether there was a difference between the happy and sad conditions within the infant conditions, and mothers were faster for recognizing happy faces than sad faces, $t(65)=-9.72, p<.001$. This was consistent with the faster recognition for happy faces than sad faces that was also present in the adult condition, $\mathrm{t}(65)=-17.98, p<.001$.

\section{Discussion}

In the current study, we investigated the speed of responding in mothers when recognizing happy or sad expressions in adult and infant faces. Mothers were faster when recognizing affect in adult faces compared to infant faces, reflecting the same own-age bias demonstrated in the general adult literature (e.g., Rhodes \& Anastasi, 2012). Mothers were also faster when recognizing happy versus sad faces in both conditions, in line with the happy-face bias observed in the general adult population (e.g., Kirita \& Endo, 1995).

Importantly however, we found an interaction between facial age and affect for reaction time, wherein the reaction time difference in happy versus sad face recognition conditions were more similar for infant faces than adult faces. These findings in mothers of children under one year old replicate the previous findings of Byrne et al. (2019) who employed the same paradigm in an independent sample of pregnant women.

As earlier discussed, motherhood may represent a time when greater sensitivity to infant cues may lead to greater attentional allocation to infant stimuli (e.g., Brosch, Sander, \& Scherer, 2007; Proverbio et al., 2006; Thompson-Booth et al., 2014a; Thompson-Booth et al., 2014b). This heightened salience to infant cues may explain the slower responding to affect in infant faces, as well as smaller discrepancy between responding to happy and sad infant faces relative to the adult face condition. Furthermore, attentional research demonstrates that there are also greater levels of attention, and greater difficulties disengaging attention from stimuli, when negative emotion is involved (e.g., Brosch et al., 2007; 
Eastwood, Smilek, \& Merikle, 2003). In the current study, a similar pattern of responding can be observed in Figure 2 where maternal reaction times were slowest for the sad infant face condition. Thus, distressed infant faces may represent particularly salient stimuli requiring a significant allocation of attentional resources that leads to disrupted attention disengagement and redirection. This difficulty disengaging attention may then result in a slower overall reaction time, as the mother's attention may be held longer by the distressed infant expression, and she would need an effortful redirection of attention to attend to the morph task's response buttons. Given greater attentional bias to, and difficulty disengaging attention from, distressed infant expressions has been previously correlated with higher quality mother-infant relationships (Pearson, Lightman, \& Evans, 2011). Therefore, the current findings may hold value for the delivery of appropriate caregiving responses, formation of mother-healthy infant attachment, and the child's subsequent development later on. This will be an important direction for future research to further examine.

Our results can also be interpreted in the context of the broader emotion recognition literature regarding the happy face advantage and the own-age bias. The demonstrated faster responding to happy faces within both infant and adult conditions may be considered with respect to the unique facial features for happy faces. Prior research suggests that happy expressions are processed using more holistic processing approaches (Kirita \& Endo, 1995). The happy expressions in both age conditions featured smiles that may have led to increased ability and speed in recognizing this unique feature (Adolphs, 2002), allowing for a faster response time. Conversely, sad expressions have been found to be processed in a more analytic manner (Kirita \& Endo, 1995) given their greater comparative complexity, which therefore slows reaction time and may explain the slower reaction time to sad faces in both conditions. The happy face advantage findings are unlikely to be explained by intensity differences between each emotional expression condition given that sad faces were rated as more intense than happy faces (counter to what would be predicted for a happy face advantage). In combination with the own- age bias, in which recognition is superior for faces within same-age group, we may presume that the cumulative effect of both a happy expression as well as a same-aged expression resulted in the fastest reaction time for adult happy expressions. 
There were a number of limitations of the current study. Despite its use in previous studies, the facial morph task may not be reflective of real-life experiences that require the recognition of age and affect, and may be oversimplified. It is important to note that given the very nature of the stimulus set, infant facial expressions may appear more naturalistic and adults expressions more posed; however, we did not see this reflected in intensity ratings of infant as compared to adult faces suggesting their comparability in this dimension. The facial morph task's previous limitation, as noted by Byrne et al. (2019), still stands in that it only includes Caucasian faces as stimuli. Although no correlation between ethnicity and processing performance was found in the current study, it is of consequence that the current study collected information only in terms of whether mothers identified as Hispanic or Non-Hispanic. Racial differences have been noted in other studies of the own-race versus other-race impact (e.g., Hodsoll, Quinn, \& Hodsoll, 2010; Lee et al., 2008), but our demographic information may not have been detailed enough to thoroughly investigate this potential processing bias. Furthermore, the socioeconomic status of our sample (as determined by education) was moderately high, which may limit the generalizability of our findings to a more socioeconomically diverse group.

It may also stand that the current facial morphing paradigm is more useful for establishing general patterns of behavior, and is not as sensitive for delineating differences between individuals. This possible limitation may serve as an explanation in regard to our null findings for the impact of various demographic variables, as well as both anxiety and depression symptoms, on task performance. Furthermore, although the current study focused only on a sample of mothers, caregiving behaviors apply to both mothers and fathers. It may prove useful to examine whether or not fathers perform similarly on the facial morphing task in further research. Finally, it may be of worth to examine if there are differences between parental performance and non-parental performance on the morphing paradigm in future iterations of the task, including careful consideration of potential confounding sociodemographic factors. While this will provide important insight into the maternal specificity of the findings, and its exclusion may be considered a limitation of the current design, our main goal of the 
current study was to examine the replicability of facial emotion recognition performance previously observed prenatally to a postpartum sample.

\section{Conclusions}

The current study reinforces that both the own-age bias and the happy face advantage are robust in the maternal postpartum period. Furthermore, the replication of Byrne et al. (2019)'s findings further suggests the use of the current methodology as a reliable tool in examining facial emotion recognition. Understanding and continuing to investigate these processes is relevant to parenthood in particular due to the necessity of facial processing in appropriate caregiving behaviors. The current study raises additional research queries regarding paternal emotion recognition and potential differences between parent and non-parent samples. The answering of these questions, as well as others, will continue to contribute to a growing body of knowledge on the way in which cognitive and affective processes impact maternal behavior and communication with their infant, and thus the development of the child overall.

\section{Acknowledgements}

We would like to thank all the women who participated in this study through the Yale Baby School and Dr. Cynthia Willner for statistical consultation. 
References

Adolphs, R. (2002). Neural systems for recognizing emotion. Current opinion in neurobiology, 12(2), 169-177. doi:https://doi.org/10.1016/S0959-4388(02)00301-X

Ainsworth, M. S. (1979). Infant-mother attachment. American psychologist, 34(10), 932. doi:https://doi.org/10.1037/0003-066X.34.10.932

Barba-Müller, E., Craddock, S., Carmona, S., \& Hoekzema, E. (2019). Brain plasticity in pregnancy and the postpartum period: links to maternal caregiving and mental health. Archives of women's mental health, 1-11. doi:https://doi.org/10.1007/s00737-018-0889- z

Bowlby, J. (1997). Attachment (Vol. 1): Random House.

Brosch, T., Sander, D., \& Scherer, K. R. (2007). That baby caught my eye... attention capture by infant faces. doi: $\underline{\text { https://doi.org/10.1037/1528-3542.7.3.685 }}$

Byrne, S. P., Mayo, A., O’Hair, C., Zankman, M., Austin, G. M., Thompson-Booth, C., . .

Rutherford, H. J. (2019). Facial emotion recognition during pregnancy: Examining the effects of facial age and affect. Infant Behavior and Development, 54, 108-113. doi:https://doi.org/10.1016/i.infbeh.2018.09.008

Cohen, J. (1988). Statistical Power Analysis for the Behavioral Sciences, (L. Erlbaum Associates, Hillsdale, NJ): Erlbaum Associates Hillsdale, NJ.

Darwin, C., \& Prodger, P. (1998). The expression of the emotions in man and animals: Oxford University Press, USA.

Eastwood, J. D., Smilek, D., \& Merikle, P. M. (2003). Negative facial expression captures attention and disrupts performance. Perception \& psychophysics, 65(3), 352-358. doi:https://doi.org/10.3758/BF03194566

Endendijk, J. J., Spencer, H., van Baar, A. L., \& Bos, P. A. (2018). Mothers' neural responses to infant faces are associated with activation of the maternal care system and observed intrusiveness with 
their own child. Cognitive, Affective, \& Behavioral Neuroscience, 18(4), 609-621. doi:https://doi.org/10.3758/s13415-018-0592-6

Ferrey, A. E., Santascoy, N., McCrory, E. J., Thompson-Booth, C., Mayes, L. C., \& Rutherford,

H. J. (2016). Motivated attention and reward in parenting. Parenting, 16(4), 284-301. doi:https://doi.org/10.1080/15295192.2016.1184928

Fridlund, A. J. (1991). Evolution and facial action in reflex, social motive, and paralanguage. Biological psychology, 32(1), 3-100. doi:https://doi.org/10.1016/0301-0511(91)90003-Y

Glocker, M. L., Langleben, D. D., Ruparel, K., Loughead, J. W., Gur, R. C., \& Sachser, N.

(2009). Baby schema in infant faces induces cuteness perception and motivation for caretaking in adults. Ethology, 115(3), 257-263. doi:https://doi.org/10.1111/i.1439- 0310.2008.01603.x

Goldberg, S., Grusec, J. E., \& Jenkins, J. M. (1999). Confidence in protection: Arguments for a narrow definition of attachment. Journal of Family Psychology, 13(4), 475. doi:https://doi.org/10.1037/0893-3200.13.4.475

Hodsoll, J., Quinn, K. A., \& Hodsoll, S. (2010). Attentional prioritization of infant faces is limited to own-race infants. PLoS ONE, 5(9), e12509. doi:https://doi.org/10.1371/journal.pone.0012509

Hoekzema, E., Barba-Müller, E., Pozzobon, C., Picado, M., Lucco, F., García-García, D., . . Crone, E. A. (2017). Pregnancy leads to long-lasting changes in human brain structure. Nature Neuroscience, 20(2), 287. doi:http://dx.doi.org/10.1038/nn.4458

Johnston, P. J., Katsikitis, M., \& Carr, V. J. (2001). A generalised deficit can account for problems in facial emotion recognition in schizophrenia. Biological psychology, 58(3), 203-227. doi:https://doi.org/10.1016/S0301-0511(01)00114-4

Joormann, J., \& Gotlib, I. H. (2006). Is this happiness I see? Biases in the identification of emotional facial expressions in depression and social phobia. Journal of abnormal psychology, 115(4), 705.

Kim, P. (2016). Human maternal brain plasticity: Adaptation to parenting. New Directions for Child And Adolescent Development, 2016(153), 47-58. doi:https://doi.org/10.1002/cad.20168 
Kim, P., Strathearn, L., \& Swain, J. E. (2016). The maternal brain and its plasticity in humans. Hormones and behavior, 77, 113-123. doi:https://doi.org/10.1016/j.yhbeh.2015.08.001

Kirita, T., \& Endo, M. (1995). Happy face advantage in recognizing facial expressions. Acta psychologica, 89(2), 149-163. doi:https://doi.org/10.1016/0001-6918(94)00021-8

Kroenke, K., Spitzer, R. L., \& Williams, J. B. (2003). The Patient Health Questionnaire-2: validity of a two-item depression screener. Medical care, 1284-1292. doi:https://www.jstor.org/stable/3768417

Kuefner, D., Macchi Cassia, V., Picozzi, M., \& Bricolo, E. (2008). Do all kids look alike?

Evidence for an other-age effect in adults. Journal of Experimental Psychology: Human Perception and Performance, 34(4), 811. doi:https://doi.org/10.1037/0096-

\section{$\underline{1523.34 .4 .811}$}

Lee, K.-U., Khang, H. S., Kim, K.-T., Kim, Y.-J., Kweon, Y.-S., Shin, Y.-W., . . Chae, J.-H.

(2008). Distinct processing of facial emotion of own-race versus other-race. Neuroreport, 19(10), 10211025. doi:https://doi.org/10.1097/WNR.0b013e3283052df2

Lobmaier, J. S., Sprengelmeyer, R., Wiffen, B., \& Perrett, D. I. (2010). Female and male responses to cuteness, age and emotion in infant faces. Evolution and Human Behavior, 31(1), 16-21. doi:https://doi.org/10.1016/j.evolhumbehav.2009.05.004

Lorenz, K. (1943). Die angeborenen formen möglicher erfahrung. Zeitschrift für Tierpsychologie, 5(2), 235-409. doi: https://doi.org/10.1111/j.1439- 0310.1943.tb00655.x

Lorenz, K. (1971). Studies in animal and human behaviour., 281-322. doi:http://dx.doi.org/10.4159/harvard.9780674430389

Lowyck, B., Luyten, P., Vanwalleghem, D., Vermote, R., Mayes, L. C., \& Crowley, M. J. (2016). What's in a face? Mentalizing in borderline personality disorder based on dynamically changing facial expressions. Personality Disorders: Theory, Research, and Treatment, 7(1), 72.

Matlin, M., \& Stang, D. (1978). Pollyanna principle. Psychology Today, 11(10), 56. 
Mayes, L., Rutherford, H., Suchman, N., \& Close, N. (2012). The neural and psychological dynamics of adults' transition to parenthood. Zero to three, 33(2), 83. doi:https://www.ncbi.nlm.nih.gov/pubmed/23616691

McElwain, N. L., \& Booth-LaForce, C. (2006). Maternal sensitivity to infant distress and nondistress as predictors of infant-mother attachment security. Journal of Family Psychology, 20(2), 247. doi:https://doi.org/10.1037/0893-3200.20.2.247

Pearson, R. M., Lightman, S. L., \& Evans, J. (2011). Attentional processing of infant emotion during late pregnancy and mother-infant relations after birth. Archives of women's mental health, 14(1), 2331.

Proverbio, A. M., Brignone, V., Matarazzo, S., Del Zotto, M., \& Zani, A. (2006). Gender and parental status affect the visual cortical response to infant facial expression.

Neuropsychologia, 44(14), 2987-2999. doi:https://doi.org/10.1016/j.neuropsychologia.2006.06.015

Purhonen, M., Kilpeläinen-Lees, R., Pääkkönen, A., Yppärilä, H., Lehtonen, J., \& Karhu, J. (2001). Effects of maternity on auditory event-related potentials to human sound. Neuroreport, 12(13), 2975-2979. doi:https://www.ncbi.nlm.nih.gov/pubmed/11588614

Rhodes, M. G., \& Anastasi, J. S. (2012). The own-age bias in face recognition: a meta-analytic and theoretical review. Psychological Bulletin, 138(1), 146.

doi:https://doi.org/10.1037/a0025750

Schneider, W., Eschman, A., \& Zuccolotto, A. (2012). E-Prime 2.0 reference guide manual.

Pittsburgh, PA: Psychology Software Tools.

Seifritz, E., Esposito, F., Neuhoff, J. G., Lüthi, A., Mustovic, H., Dammann, G., . . Tedeschi, G. (2003). Differential sex-independent amygdala response to infant crying and laughing in parents versus nonparents. Biological psychiatry, 54(12), 1367-1375. doi:https://doi.org/10.1016/S0006$\underline{3223(03) 00697-8}$ 
Snyder, R., Shapiro, S., \& Treleaven, D. (2012). Attachment theory and mindfulness. Journal of Child and Family Studies, 21(5), 709-717. doi:https://doi.org/10.1007/s10826-011- 9522-8

Spitzer, R. L., Kroenke, K., Williams, J. B., \& Löwe, B. (2006). A brief measure for assessing generalized anxiety disorder: the GAD-7. Archives of internal medicine, 166(10), 1092- 1097. doi:https://doi.org/10.1001/archinte.166.10.1092

Strathearn, L., \& McClure, S. (2002). A functional MRI study of maternal responses of infant facial cues. Paper presented at the Annual scientific meeting of the Society for Neuroscience, abstract viewer.

Thompson-Booth, C., Viding, E., Mayes, L. C., Rutherford, H. J., Hodsoll, S., \& McCrory, E. (2014a). I can't take my eyes off of you: attentional allocation to infant, child, adolescent

and adult faces in mothers and non-mothers. PLOS ONE, 9(10), e109362. doi:https://doi.org/10.1371/journal.pone.0109362

Thompson-Booth, C., Viding, E., Mayes, L. C., Rutherford, H. J., Hodsoll, S., \& McCrory, E. J. (2014b). Here's looking at you, kid: Attention to infant emotional faces in mothers and non-mothers. Developmental science, 17(1), 35-46. doi:https://doi.org/10.1111/desc.12090

Tottenham, N., Tanaka, J. W., Leon, A. C., McCarry, T., Nurse, M., Hare, T. A., . . Nelson, C. (2009). The NimStim set of facial expressions: judgments from untrained research participants. Psychiatry research, 168(3), 242-249. doi:https://doi.org/10.1016/j.psychres.2008.05.006

Valentine, T. (1991). A unified account of the effects of distinctiveness, inversion, and race in face recognition. The Quarterly Journal of Experimental Psychology Section A, 43(2), 161-204. doi:https://doi.org/10.1080/14640749108400966

\section{Author Statement}

Madison Bunderson: Formal Analysis, Project Administration, Investigation, Writing - Original Draft Kathryn Armstrong: Investigation, Project Administration, Writing - Reviewing and Editing Linda Mayes: Conceptualization, Methodology, Resources, Project Administration, Writing - Reviewing and Editing 
Eamon McCrory: Conceptualization, Methodology, Resources, Writing - Reviewing and Editing Chloe Thompson-Booth: Conceptualization, Methodology, Resources, Writing - Reviewing and Editing

Helena Rutherford: Conceptualization, Methodology, Formal Analysis, Investigation, Writing Reviewing and Editing, Project Administration, Supervision 